\title{
Learning-Specific Changes in Long-Term Depression in Adult Perirhinal Cortex
}

\author{
Peter V. Massey, Daniel Phythian, Katherine Narduzzo, Elizabeth C. Warburton, Malcolm W. Brown, and \\ Zafar I. Bashir \\ Medical Research Council Centre for Synaptic Plasticity, Department of Anatomy, University of Bristol, Bristol BS8 1TD, United Kingdom
}

Learning is widely believed to involve synaptic plasticity, using mechanisms such as those used in long-term potentiation (LTP). We assess whether the mechanisms used in alternative forms of plasticity, long-term depression (LTD) and depotentiation, play a role in learning. We have exploited the involvement of the perirhinal cortex in two different forms of learning to compare simultaneously, within the same brain region, their effects on LTD and depotentiation. Multiple-exposure learning but not single-exposure learning in vivo prevented, in a muscarinic receptor-dependent manner, subsequent induction of LTD and depotentiation, but not LTP, in perirhinal cortex in vitro. The contrast in the effects of the two types of learning under these particular experimental conditions indicate that the in vitro change is unlikely to be attributable to synapse-specific plastic changes registering the precise details of the individual learned associations. Instead, it is concluded that the lack of LTD and depotentiation arises from, and establishes the importance of, a learningrelated generalized change in plasticity gain. The existence of this additional mechanism has important implications for interpretations of how plasticity relates to learning.

Key words: perirhinal cortex; synaptic plasticity; LTD; depotentiation; learning; memory

\section{Introduction}

Although learning is widely believed to depend on synaptic plasticity (Bliss and Collingridge, 1993; Martin and Morris, 2002; Abraham and Robins, 2005), the relationships between learning and synaptic plasticity are still not well understood. Mechanisms of synaptic plasticity, notably long-term potentiation (LTP) and long-term depression (LTD), are chiefly studied in vitro (Kemp and Bashir, 2001; Malenka and Bear, 2004). Accordingly, it is important to establish links between learning and such plasticity. One way to do so is by studying in vitro synaptic plasticity after learning. Indeed, there is evidence that learning is associated with LTP-like increases in synaptic strength in motor cortex, amygdala, and hippocampus (Rioult-Pedotti et al., 1998; Rodrigues et al., 2004; Maren, 2005). However, there is a growing realization that LTD-like processes, including depotentiation (the reversal of LTP), may be equally effective at storing information that is essential for learning and memory (Kemp and Manahan-Vaughan, 2007; Massey and Bashir, 2007; Griffiths et al., 2008). The extent to which LTD and depotentiation, which may have different mechanisms of induction and expression (Kemp and Bashir, 2001), play different roles in learning and memory is still unclear.

Perirhinal cortex is necessary for the single-exposure learning (Brown and Aggleton, 2001; Warburton et al., 2003) of recognition memory (familiarity discrimination). Such learning is associated with long-term reductions in neuronal responsiveness

Received Dec. 18, 2006; revised June 5, 2008; accepted June 19, 2008

This work was supported by the Wellcome Trust. We are grateful to B. Fry and J. Robbins for technical assistance. Correspondence should be addressed to Prof.Zafarl. Bashir at the above address. E-mail:z.i.bashir@bristol.ac.uk. D0I:10.1523/JNEUROSCI.1935-08.2008

Copyright $\odot 2008$ Society for Neuroscience $\quad$ 0270-6474/08/287548-07\$15.00/0
(Zhu et al., 1996; Brown and Xiang, 1998; Xiang and Brown, 1998), raising the possibility that LTD-like mechanisms may underlie perirhinal cortex-dependent learning and memory (Brown and Bashir, 2002). In addition to its role in familiarity discrimination, perirhinal cortex is also necessary for multitrial perceptual and reinforcement learning (Murray and Bussey, 1999; Brown and Aggleton, 2001; Murray and Richmond, 2001). This therefore provides an opportunity to examine, in the same brain region at the same time, the effects of both forms of learning on synaptic plasticity.

To compare the effects of these two forms of learning under controlled conditions, we used a paired viewing apparatus (Wan et al., 1999, Warburton et al., 2003) in which a rat's left eye saw stimuli presented to the left while its right eye saw stimuli presented to the right. Novel pictures, to one eye, and familiar pictures, to the other, were shown to the monocular field of each eye so that the information was initially sent to the contralateral hemisphere (termed the "novel" or "familiar" hemisphere). Stimuli were shown simultaneously to separate eyes under the same behavioral conditions, so controlling for movement, alertness, brain temperature, reinforcement, emotional, hormonal, and stress levels. Picture presentations were accompanied by juice so that the rat maintained its head in a constant viewing position. Consequently, there was opportunity across trials for the repeated familiar, but not the singly presented novel, pictures to become associated with reward delivery. For each rat, perirhinal slices were prepared from "novel" and "familiar" hemispheres $60 \mathrm{~min}$ after the last presentations. Extracellular recording was used to assess synaptic transmission and plasticity (Ziakopoulos et al., 1999; Massey et al., 2004) in slices from the familiar and the novel hemispheres. 


\section{Materials and Methods}

Adult male pigmented Dark Agouti rats (170-220 g; Bantin and Kingman) were maintained on a $14 \mathrm{~h} \mathrm{light} / 10 \mathrm{~h}$ dark cycle (dark phase during normal daylight). All efforts were made to minimize animal suffering, and experiments were performed in accordance with the UK Animals (Scientific Procedures) Act (1986) and had received ethical approval.

Paired viewing. Novel and familiar pictures were presented to each rat simultaneously using the paired viewing procedure (Warburton et al., 2003 ) illustrated in Figure 1a. The apparatus consisted of a chamber $(30 \times 30 \times 35 \mathrm{~cm})$, the bottom and sides of which were black, the front made of transparent Perspex with a central observing hole $3 \mathrm{~cm}$ in diameter and $6 \mathrm{~cm}$ above the floor. The top of the chamber was open. On either side of the observing hole were two barriers that maintained the rat's body at $90^{\circ}$ to the front screen when its head was placed in the observing hole. When the rat's head was placed in the observing hole, an infrared beam was broken, signaling a computer to commence a trial. After an interval (3-4 s), provided that the head remained in the hole, two pictures $(15 \times 12 \mathrm{~cm})$ were shown simultaneously, on two separate monitors placed $30 \mathrm{~cm}$ from the observing holes. The pictures were colored two-dimensional representations of single objects (Microsoft ClipArt). A central partition ensured that each picture was seen by only one eye. In this way, novel and familiar stimuli could be shown under the same conditions of alertness and motivation and with similar eye movements. The pictures appeared for $4 \mathrm{~s}$, after which a drop of diluted blackcurrant juice was delivered by a metal tube that the rat could reach and lick. Each presentation of pictures was followed by delivery of juice. Experiments were video recorded so that behavior and position of the animals head and body could be monitored. The experiments were counterbalanced so that for some animals it was the left and for some animals it was the right eye that saw novel stimuli, and vice versa for familiar stimuli.

Training. During training, rats had ad libitum access to water for $2 \mathrm{~h}$ on each day. Each rat was pretrained over $2 \mathrm{~d}$ to go to the observing hole for a juice reward (no stimulus presentation). Training consisted of four single sessions, one per day. On each day, one eye was exposed to a new set of 60 novel stimuli, while the other eye viewed the same set of 15 stimuli, four times. Thus, by the final training day, one eye had been exposed to 16 times more novel stimuli than the other, but each eye was exposed to the same number (240) of stimuli. Control animals were treated in the same way except that the computer monitors were switched off, and therefore the control animals were not shown visual stimuli. Where used, scopolamine hydrobromide or scopolamine methylbromide (methyl scopolamine; Sigma Chemicals) was given systemically intraperitoneally at $0.05 \mathrm{mg} / \mathrm{kg}$ in a volume of $1 \mathrm{ml} / \mathrm{kg}$ normal saline 30 min before each training session.

Slice preparation and electrophysiology. One hour after presentation of the final stimuli, animals were anesthetized with an isoflurane/oxygen mixture and decapitated, and the brain was rapidly removed. The brain was placed in ice-cold artificial CSF (aCSF; bubbled with $95 \% \mathrm{O}_{2} / 5 \%$ $\mathrm{CO}_{2}$ ) which comprised the following (in $\mathrm{mM}$ ): $124 \mathrm{NaCl}, 3 \mathrm{KCl} ; 26$ $\mathrm{NaHCO}_{3}, 1.25 \mathrm{NaH}_{2} \mathrm{PO}_{4}, 2 \mathrm{CaCl}_{2}, 1 \mathrm{MgSO}_{4}$, and 10 D-glucose. A midsagittal section was made, the rostral and caudal parts of the brain were removed by single scalpel cuts at $\sim 45^{\circ}$ to the dorsoventral axis, and each hemisphere was glued by its caudal end to a vibroslice stage (Campden Instruments). Slices $(400 \mu \mathrm{m})$ of perirhinal cortex were taken in the region $4 \mathrm{~mm}$ behind bregma. Slices were stored submerged in aCSF $\left(20-25^{\circ} \mathrm{C}\right)$ for $1-6 \mathrm{~h}$ before transferring to the recording chamber. A single slice was placed in a submerged recording chamber $\left(28-30^{\circ} \mathrm{C}\right.$; flow rate $\sim 2 \mathrm{ml} / \mathrm{min}$ ) when required. Slices from each hemisphere were kept separate with the experimenter blind to the hemispheric origin of the slices.

Standard in vitro extracellular field recordings were made from the perirhinal cortex (Ziakopoulos et al., 1999; Massey et al., 2004). Evoked field EPSPs (fEPSPs) were recorded from layers II/III from directly below the rhinal sulcus (area 35). Two stimulating electrodes were placed on both sides $(\sim 0.5 \mathrm{~mm})$ of the recording electrode and designated entorhinal input (area 35) and temporal input (area 36). Stimuli (constant voltage) were delivered alternately to the two stimulating electrodes
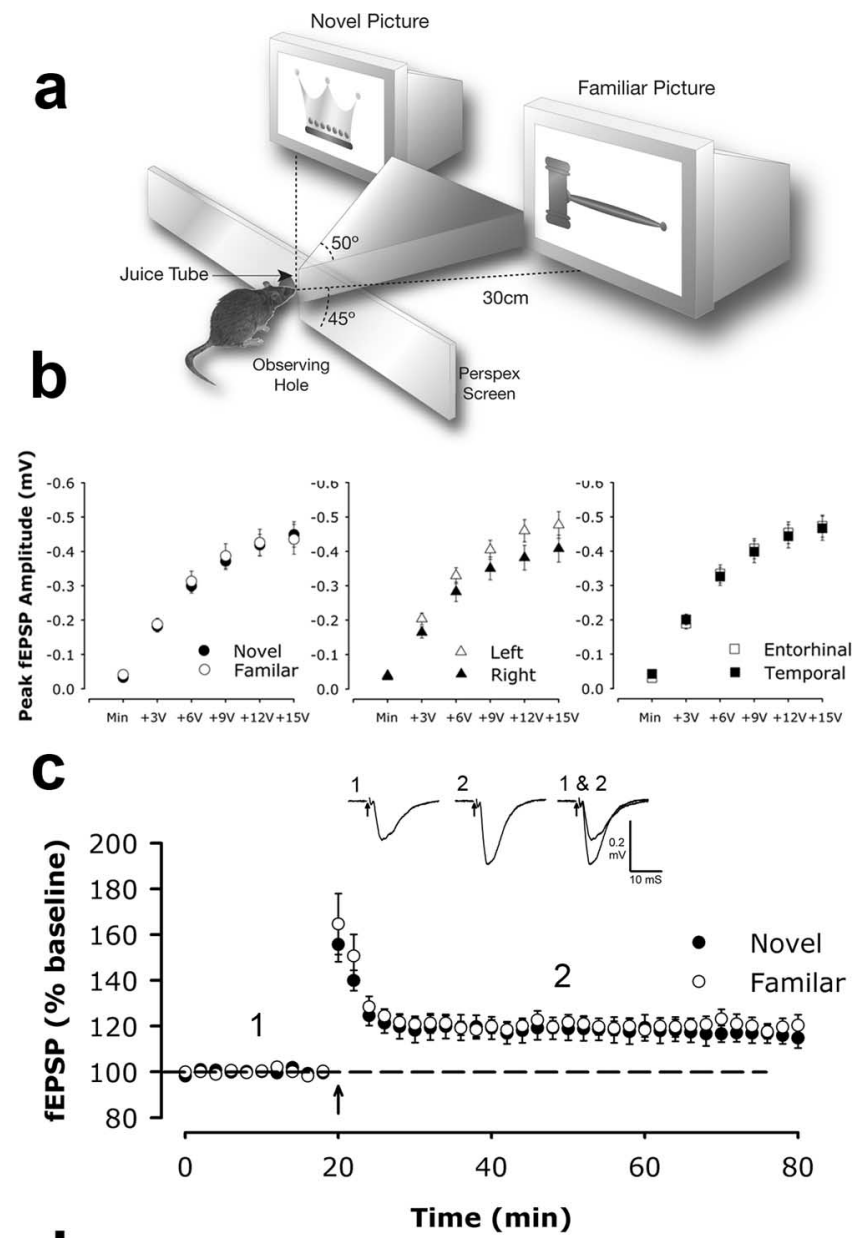

d

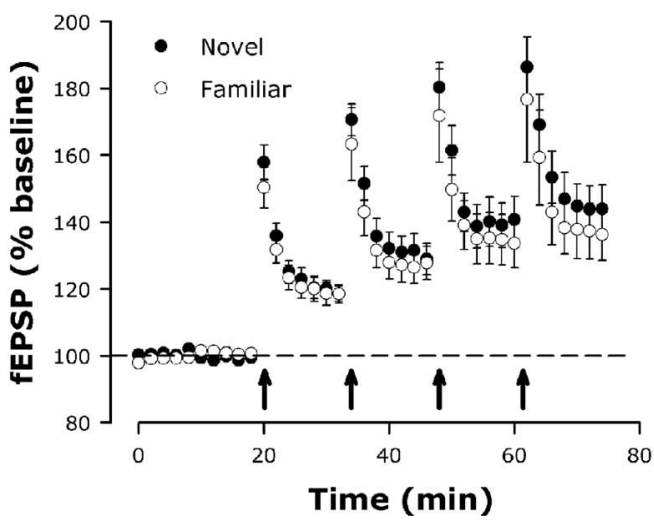

Figure 1. Lack of effect of viewing novel or familiar stimuli on synaptic transmission or LTP. $\boldsymbol{a}$, The paired viewing apparatus. The rat receives a juice reward for keeping its head in the observing hole. Pictures, novel on one side and familiar on the other, are shown simultaneously on two monitors, each visible to only one eye. In this way, information for novel stimuli is primarily delivered to one hemisphere ("novel"), and information for familiar stimuli is primarily delivered to the other ("familiar"). $\boldsymbol{b}$, There is no difference in the input/output characteristics of fEPSPs when assessed as a function of novel versus familiar (left; $p>0.05 ; n=13$ ); right versus left hemisphere (middle; $p>0.05 ; n=12$ ); or comparison of the two stimulating electrodes (entorhinal vs temporal; right; $p>0.05 ; n=12$ ). The graphs illustrate increasing stimulus intensity versus synaptic strength. $c$, Pooled data showing that LTP that lasts at least $1 \mathrm{~h}$ is induced by HFS $(100 \mathrm{~Hz}, 1 \mathrm{~s}$ stimulation, repeated 4 times, as indicated by the upward arrow). There is no difference in the magnitude of LTP between novel and familiar hemispheres $(p>0.05 ; n=7)$. d, Repeated HFS ( 1 train of $100 \mathrm{~Hz}$ every $14 \mathrm{~min}$ ) saturates LTP. Data show that there is no difference in the magnitude of potentiation between novel and familiar hemispheres ( $p>0.05 ; n=11$; measured 15 min after HFS). In this and subsequent figures, the heterosynaptic pathway is not shown for the sake of clarity. Error bars represent SEM. 
(each electrode $0.033 \mathrm{~Hz}$ ). Two pathways were examined to ensure input specificity of any plasticity effects, as previously described (Ziakopoulos et al., 1999; Massey et al., 2004). Input/output curves were produced by stimulating initially at "minimal" intensity (sufficient to produce an fEPSP discernable from the noise) and increased intensities in $3 \mathrm{~V}$ steps until a maximal fEPSP was achieved (Fig. 1). The minimal intensity across experiments ranged between 2 and $4 \mathrm{~V}$. fEPSPs were reduced to $50-60 \%$ of maximum amplitude to achieve a baseline of synaptic transmission before induction of synaptic plasticity. After a baseline of at least $30 \mathrm{~min}$, high-frequency stimulation (HFS; four trains, each of $100 \mathrm{~Hz}, 1 \mathrm{~s}$, every $15 \mathrm{~s}$ ) was delivered to induce LTP. Subsequently, to induce depotentiation, low-frequency stimulation ( 900 stimuli, $1 \mathrm{~Hz}$ ) was applied 20 min after LTP induction. To induce de novo synaptic LTD, $5 \mathrm{~Hz}$ stimulation (10 min train) was delivered (Massey et al., 2004). Field potentials were recorded and reanalyzed off-line (Anderson and Collingridge, 2001). The peak amplitude of evoked fEPSPs was measured and expressed relative to the preconditioning baseline. LTP was measured at either 20 or $60 \mathrm{~min}$, and depotentiation and LTD at $60 \mathrm{~min}$ after conditioning. The pathway in which plasticity was induced was randomly allocated; on some occasions, it was the entorhinal and on others, the temporal input. LTP, LTD, and depotentiation were input specific with no plasticity observed in the nonconditioned pathway (data not shown), as has previously been described (Ziakopoulos et al., 1999; Cho et al., 2000; Warburton et al., 2003; Massey et al., 2004). The significance of plasticity changes (LTP, depotentiation, LTD) was established using either paired or unpaired $t$ tests or ANOVAs with repeated measures, as appropriate.

\section{Results}

No differential effect of viewing novel or familiar stimuli on synaptic transmission or LTP

We first tested for changes in excitability of transmission and LTP. Stimulus-response characteristics of perirhinal field EPSPs in slices from novel or familiar hemispheres did not differ $(p>$ $0.05 ; n=13$ ) at any stimulus intensity tested (Fig. 1b). LTP was induced in perirhinal slices from both novel and familiar hemispheres (Fig. 1c). The magnitude of LTP, measured after $60 \mathrm{~min}$, did not differ between novel and familiar hemispheres (novel, $116 \pm 4 \%$; familiar, $119 \pm 4 \% ; p>0.05 ; n=7$ ) (Fig. $1 c$ ). Furthermore, the magnitude of potentiation induced by several periods of high-frequency stimulation (one train of $100 \mathrm{~Hz}$ every 14 min) did not differ between novel and familiar hemispheres $(p>$ $0.05 ; n=15)($ Fig. $1 d)$.

\section{Depotentiation is prevented by repeated viewing of familiar} but not by single viewing of novel stimuli

We next tested for changes in depotentiation. In contrast to the lack of effects on LTP, depotentiation of perirhinal field EPSPs differed markedly between novel and familiar hemispheres (paired $t=2.27 ; p<0.05 ; n=13$ pairs) (Fig. $2 a$ ). Depotentiation was induced by low-frequency stimulation ( 900 stimuli, $1 \mathrm{~Hz}) 20$ min after induction of LTP. In novel hemispheres, full depotentiation to baseline $(100 \%)$ was induced (LTP, $122 \pm 5 \%$; depotentiation to $100 \pm 5 \% ; p<0.05$ compared with LTP; $n=13$ ) (Fig. 2a). However, in familiar hemispheres, there was no depotentiation (LTP, $123 \pm 4 \%$; depotentiation to $118 \pm 6 \% ; p>0.05$ compared with LTP; $n=13$ ) (Fig. $2 a$ ). Normalizing to LTP levels (Fig. 2b) highlights the difference between the depotentiated novel $(84 \pm 3 \%)$ and unchanged familiar (97 $\pm 3 \%$ ) hemispheres. The sides of the brain receiving novel and familiar information were counterbalanced between animals so that any leftright bias would not affect the results. In fact, no difference in the magnitude of depotentiation was found either between novel right and left hemispheres ( $p>0.05 ; n=11$ ) or between familiar right and left hemispheres ( $p>0.05 ; n=11$; data not shown).

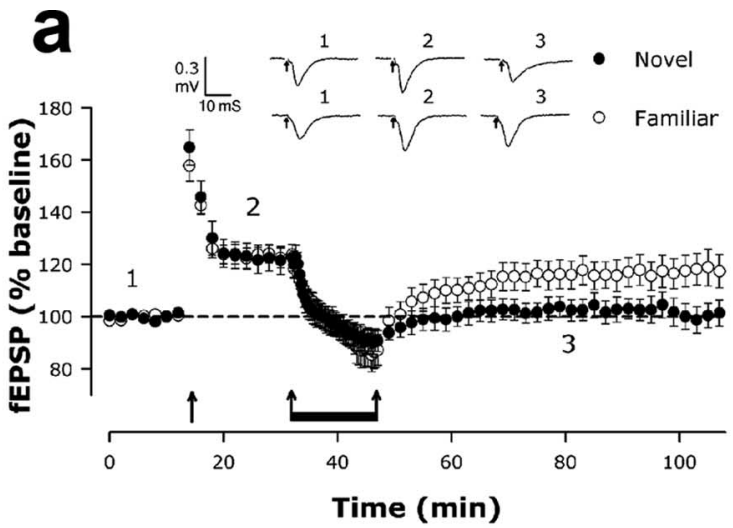

b
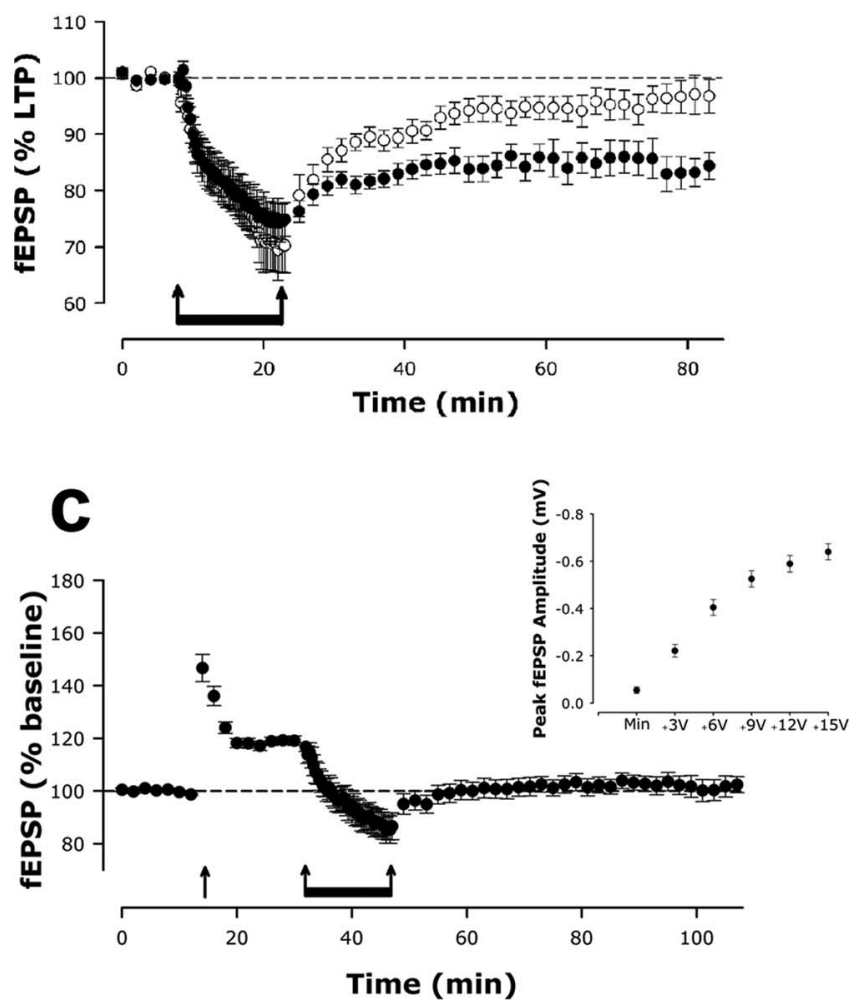

Figure 2. Induction of depotentiation is prevented in familiar hemispheres. $\boldsymbol{a}$, LTP, induced by HFS (indicated by the single upward arrow), is not different between novel and familiar hemispheres. Depotentiation, induced by $1 \mathrm{~Hz}$ stimulation for $15 \mathrm{~min}$ (indicated by the two joined arrows), occurs in novel hemispheres but not in familiar hemispheres. The traces illustrate fEPSPs taken from the time points indicated. Stimulus artifacts are blanked and replaced by arrows. $\boldsymbol{b}$, Levels of depotentiation are renormalized to LTP levels to emphasize the lack of depotentiation in familiar hemispheres compared with the depotentiation in novel hemispheres. c, LTP and depotentiation were induced in slices from control animals (paired viewing procedure, no visual stimuli). Inset, Stimulus-response characteristics for control animals. Min, "Minimal" intensity (sufficient to produce an fEPSP discernable from the noise). Error bars represent SEM.

Thus, the loss of depotentiation was only observed in familiar hemispheres, regardless of which side received this information.

LTP and depotentiation in slices from control animals

One of the advantages of the design of the experiment is that the novel and familiar hemispheres served as a within animal control for one another. However, because of the differences in depotentiation observed between the novel and familiar hemispheres, it 

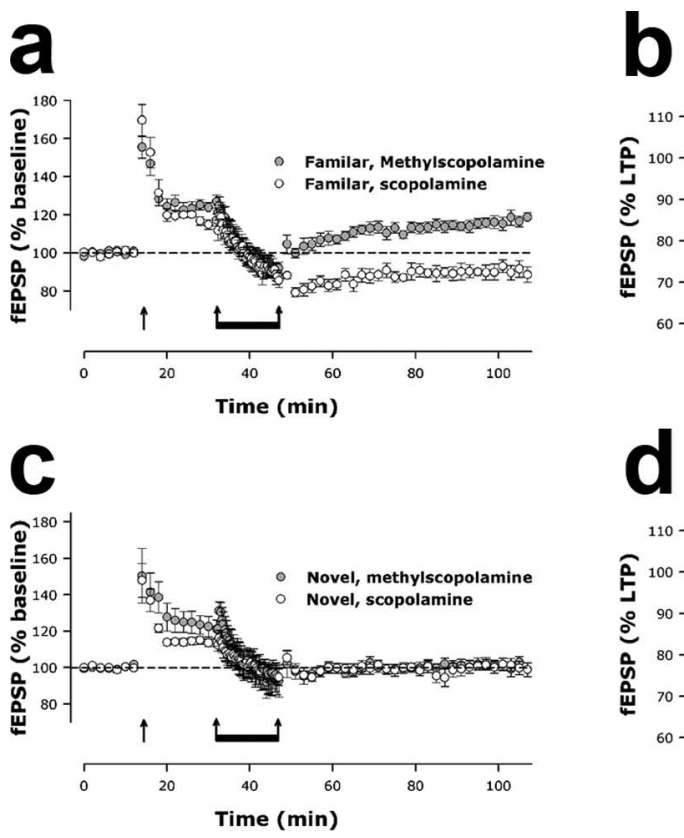

Figure 3. The loss of depotentiation by viewing familiar stimuli is dependent on muscarinic receptor activation. $\boldsymbol{a}, \boldsymbol{c}$, LTP is induced by HFS, as indicated by the single upward arrow. $\boldsymbol{a}-\boldsymbol{d}$, Depotentiation is then induced by $1 \mathrm{~Hz}$ stimulation for 15 min, as indicated by the two joined arrows. $\boldsymbol{a}$, The lack of depotentiation in familiar hemispheres when paired viewing was in the presence of methylscopolamine (controls). However, depotentiation occurred in familiar hemispheres when paired viewing was in the presence of scopolamine. $\boldsymbol{b}$, Levels of depotentiation in familiar hemispheres renormalized to LTP levels, emphasizing the difference between the effects of scopolamine and methylscopolamine treatments. $\boldsymbol{c}$, In novel hemispheres, depotentiation occurs regardless of prior treatment with scopolamine or methylscopolamine. $\boldsymbol{d}$, Levels of depotentiation in novel hemispheres renormalized to LTP levels, demonstrating the lack of difference between scopolamine and methylscopolamine treatment. Error bars represent SEM.

was important also to run additional controls. Control animals were treated in exactly the same way as those viewing novel and familiar stimuli except that blank screens were presented in the paired viewing apparatus (see Materials and Methods). Therefore, the only difference between the control animals and those viewing novel and familiar stimuli was that visual stimuli were not presented to the control animals. In slices from the control animals, stimulus-response characteristics and synaptic plasticity were examined. The stimulus-response curve was similar to those for novel and familiar hemispheres (Fig. 2c). Both LTP and depotentiation were induced in slices from control animals (LTP, $117 \pm 2 \%$; depotentiation to $102 \pm 4 \%$; $p<0.05$ for both; $n=$ 15). As previously reported (Ziakopoulos et al., 1999), there was no difference $(p>0.05)$ in the magnitude of LTP or depotentiation between entorhinal or temporal inputs (data not shown).

Thus, depotentiation occurs in perirhinal slices except when the perirhinal cortex has received information about repeated familiar stimuli.

\section{Muscarinic receptor activation is required for the prevention of depotentiation by familiar stimuli}

One explanation for the effects of learning on depotentiation is that the neuronal processes underlying the learning engage depotentiation-like mechanisms and therefore occlude the induction of further depotentiation. To test further this potential relationship, we exploited the findings that blocking acetylcholine muscarinic receptors prevents familiarity discrimination (Warburton et al., 2003; Winters and Bussey, 2005), reward learning (Aigner and Mishkin, 1993; Harder et al., 1998), and perceptual learning and its related long-term cortical changes (Wilson et al., 2004). We hence used scopolamine to block learn- ing in vivo and subsequently examined effects on depotentiation in vitro. When scopolamine $(0.05 \mathrm{mg} / \mathrm{kg}$, i.p.) was given during paired viewing, it was now possible to induce significant depotentiation in familiar hemispheres (LTP, $118 \pm 2 \%$; depotentiation to $90 \pm 4 \% ; n=6 ; p<0.05$ ) (Fig. $3 a, b)$. In contrast, in interleaved controls (using methylscopolamine, $0.05 \mathrm{mg}$ / kg, i.p., which does not cross the bloodbrain barrier), depotentiation was not observed in familiar hemispheres (LTP, $124 \pm 3 \%$; depotentiation to $118 \pm 2 \%$; $n=5$ ) (Fig. 3a,b). The difference between the magnitude of depotentiation in the scopolamine and the methylscopolamine controls was significant (unpaired $t=$ 5.38; $p<0.001 ; n=10$ ). In novel hemispheres, depotentiation was induced regardless of whether scopolamine (100 土 $3 \% ; n=5$; $p<0.05$ ) (Fig. $3 c, d$ ) or methylscopolamine $(101 \pm 4 \% ; n=5)$ (Fig. $3 c, d)$ was given. In confirmation of the finding when no drugs were used, when methylscopolamine was used the difference between the magnitude of depotentiation in the novel and familiar hemispheres was significant (unpaired $t=3.20$; $p=0.01 ; n=11)$. The magnitude of LTP or depotentiation was not dependent $(p>$ 0.05 , for both) on whether temporal or entorhinal inputs were being examined (data not shown). Thus, blocking muscarinic receptor-dependent learning prevents the loss, or occlusion, of depotentiation that occurs with repeated viewing of familiar stimuli.

\section{Multiple-trial learning prevents induction of LTD in a muscarinic receptor-dependent manner}

We next investigated learning effects on LTD. With paired viewing in the presence of methylscopolamine (control), LTD was induced in novel hemisphere slices $(17 \pm 4 \%$ depression; $p<$ $0.05 ; n=10)$ but not in familiar hemisphere slices $(6 \pm 5 \%$ depression; $p>0.05 ; n=10$ ) (Fig. $4 a$ ). The difference in LTD between the novel and familiar hemispheres was significant (paired $t=3.12 ; p=0.01 ; n=10$ pairs). Therefore, repeated presentation of familiar stimuli prevented induction of LTD. In interleaved experiments with paired viewing in scopolamine, LTD was induced in both novel $(14 \pm 4 \% ; p<0.05 ; n=12)$ and familiar $(16 \pm 3 \% ; p<0.05 ; n=12$ ) (Fig. $4 b)$ hemisphere slices. There was no difference $(p>0.05)$ in the magnitude of LTD between entorhinal and temporal inputs within the different groups (data not shown). Thus, repeated viewing of familiar, but not novel, stimuli most likely engages muscarinic receptordependent LTD-like mechanisms and therefore occludes subsequent LTD.

\section{LTD in slices from control animals}

Significant LTD was induced in slices from control animals that had experienced the paired viewing apparatus but had not been presented with visual stimuli $(17 \pm 2 \% ; p<0.05 ; n=10)$, and the magnitude of LTD was not different $(p>0.05)$ between temporal and entorhinal inputs. 


\section{a}
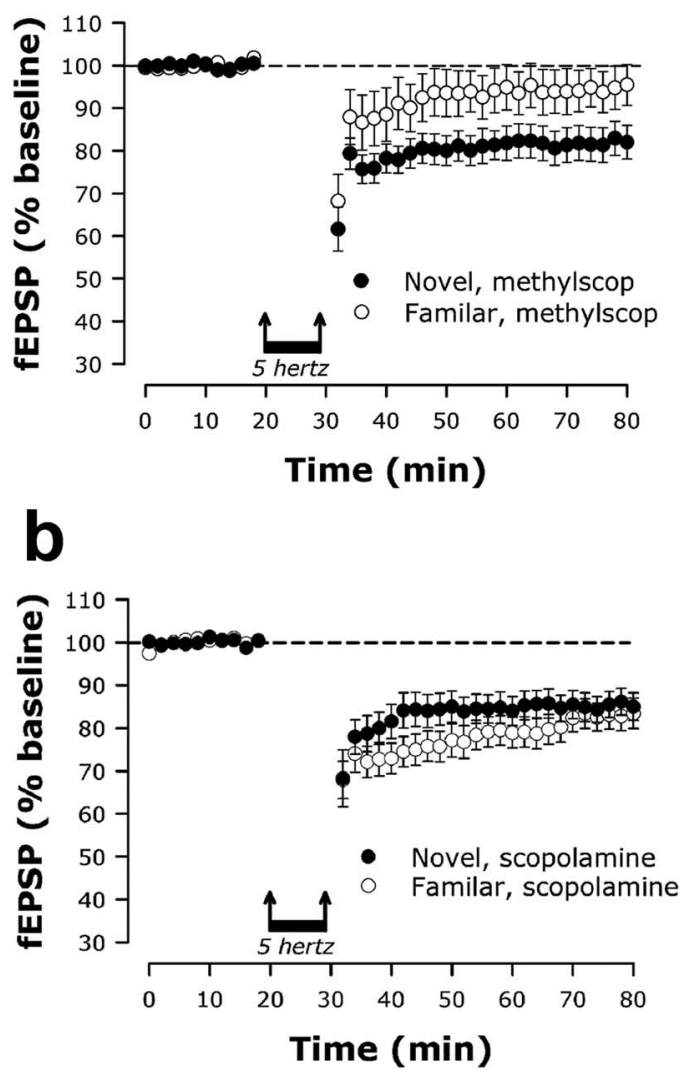

C

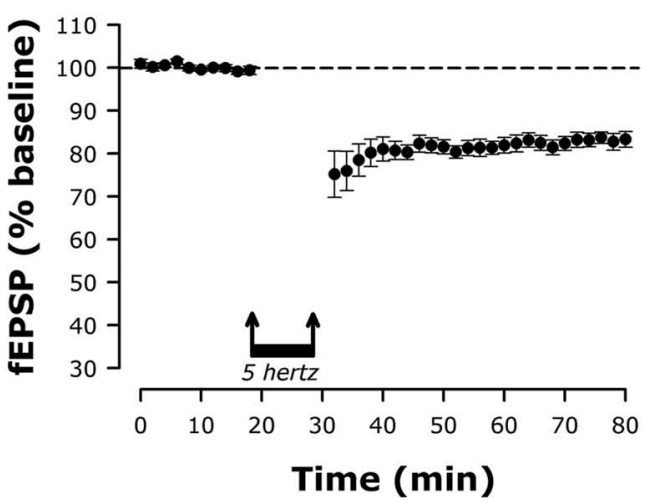

Figure 4. The prevention of LTD by prior viewing of familiar stimuli is muscarinic receptor dependent. $\boldsymbol{a}-\boldsymbol{c}$, LTD is induced by $5 \mathrm{~Hz}$ stimulation for $10 \mathrm{~min}$, as indicated by the joined arrows. $\boldsymbol{a}$, When paired viewing was performed in the presence of methylscopolamine (methylscop), LTD was induced by $5 \mathrm{~Hz}$ stimulation in novel hemispheres but was not induced in familiar hemispheres. $\boldsymbol{b}$, Paired viewing in the presence of scopolamine prevents the loss of LTD in familiar hemispheres; the level of LTD in novel hemispheres is unaffected by scopolamine pretreatment. c, LTD is induced in control animals (paired viewing procedure, no visual stimuli).

\section{Discussion}

The results demonstrate unequivocally that visual experience produces changes in synaptic plasticity in adult perirhinal cortex. These changes were an occlusion of LTD and depotentiation but no occlusion of LTP. Moreover, baseline transmission was unchanged so that the change was in underlying synaptic plasticity rather than in synaptic transmission. Importantly, the effect on plasticity was found for multiple-exposure learning (perceptual or reward association learning induced by repeated presentations of familiar stimuli) but not for single-exposure learning (learning related to recognition memory produced by single presentations of novel stimuli). The contrast is dramatic because these two types of learning were occurring simultaneously in the left and right perirhinal cortices under the same behavioral conditions. Our study is the first to compare directly changes related to these different types of learning. Furthermore, it highlights the potential importance of synaptic plasticity-like mechanisms in learning and memory.

That the interaction or cross-over of information between the hemispheres is small is established by previous studies of the Fos difference between the hemisphere receiving novel and that receiving familiar information (Zhu et al., 1996; Xiang and Brown, 1998; Warburton et al., 2003, 2005). In the current study, the difference in the effects on plasticity in the two hemispheres further confirms the relative independence of the two hemispheres.

To exclude artifactual generation of the plasticity differences, the comparison between the two types of learning was made using very closely controlled conditions. First, the use of the paired viewing procedure ensured that the two sets of stimuli (each using the same overall number of stimulus presentations) were seen simultaneously under the same behavioral conditions of movements, alertness, body temperature, reinforcement, emotional, hormonal, and stress levels. Second, the hemispheres that received novel and familiar information were counterbalanced; thus, for half the animals, the novel hemisphere was the left, and for the other half, the right. Third, preparation of perirhinal cortex from left and right hemispheres was randomized to ensure there was no bias with regard to whether novel or familiar hemispheres were dissected, sliced, or investigated first. Fourth, the experimenter carrying out the electrophysiology was blind to the history of the left and right hemisphere; the code was broken only after analysis of the individual slice data had been completed. Fifth, depotentiation and LTD were induced in perirhinal slices from control groups of animals that were trained in the apparatus but with no visual stimuli being presented. These controls argue against exposure to the apparatus alone causing loss of plasticity that is reversed by presentation of novel stimuli. An additional argument against the artifactual generation of the lack of LTD and depotentiation is that they were blocked if the visual experience was given in the presence of the muscarinic antagonist scopolamine (but not methylscopolamine, which does not cross the blood-brain barrier). Thus, the loss of LTD and depotentiation relies on the presentation of familiar visual stimuli in a muscarinic receptor-dependent manner.

We have previously reported that there are no differences in long-term plasticity between temporal and entorhinal inputs into perirhinal cortex (Ziakopoulos et al., 1999; Cho et al., 2000). Similarly, in the current study, we found no differences between the two inputs with regard to LTP, LTD, or depotentiation. Thus, the differences that exist in GABAergic transmission between the feedforward temporal and feedback entorhinal inputs (Ziakopoulos et al., 2000; Garden et al., 2002) do not significantly impact LTP, LTD, or depotentiation.

Recognition memory is a form of learning that occurs with a single exposure to a novel stimulus. In contrast, perceptual or reinforcement learning requires multiple exposures. In the standard view, both types of learning should result in synapse-specific plastic changes that store the details of the specific learning associations/occurrences; however, only one hemisphere showed an in vitro change. The observed plasticity changes are large in the familiar hemisphere and in other studies of multiple-exposure learning (Rioult-Pedotti et al., 1998; Xu et al., 1998; Manahan- 
Vaughan and Braunewell, 1999; Abraham et al., 2002; Martin and Morris, 2002; Rodrigues et al., 2004; Maren, 2005; Gruart et al., 2006). Others have raised the issue of whether the networks involved would have the capacity to store many such changes, if the observed change is a true reflection of the proportion of synapses encoding the learning of a specific, single association (Shors and Matzel, 1997; Martin and Morris, 2002; Abraham and Robins, 2005). Presuming that changes at individual synapses storing the specific, individual learned associations/occurrences occur in both hemispheres, the contrast between the hemispheres in our results strongly suggests that the changes seen after multitrial reinforcement learning arise from generalized alterations in the plasticity gain of pathways, perhaps reflecting nonspecific, salience-related effects (Shors and Matzel, 1997). In such case and in contrast, any generalized learning-related change evoked by single-exposure learning in the novel hemisphere was too small to be detected. In confirmation of this finding that plasticity changes after single-trial learning are small are the findings of a study (Whitlock et al., 2006) of single-trial inhibitory avoidance learning. In that study, changes in in vivo LTP were found at a small proportion of sampled sites and were not statistically significant in averages across all sites. Our results now indicate that additional evidence will be required to establish that the reported LTP changes did not result from a weak generalized learning-related change.

Interestingly, the results of this present study indicate that repeated viewing of familiar stimuli most likely relies on muscarinic receptor-dependent mechanisms. Furthermore, this muscarinic receptor-dependent learning mechanism prevents subsequent LTD and depotentiation but not LTP. One simple conclusion that may be drawn from these findings is that the synaptic mechanisms that are engaged during this form of learning are muscarinic receptor dependent and LTD-like, as has been suggested previously (Warburton et al., 2003; Griffiths et al., 2008). Despite observing no effects on LTP in the current study, it is still possible that LTP-like mechanisms operate in familiarity discrimination. Indeed, interfering with mechanisms that block LTP can block familiarity discrimination (Warburton et al., 2005). Although viewing of familiar stimuli resulted in an interaction with LTD and depotentiation, there was no effect observed on basal synaptic transmission. This suggests that if familiarity discrimination is associated with the induction of synaptic depression, then either this is not of sufficient magnitude to be observed under the conditions of our experiments or indeed homeostatic LTP might also have occurred to "reset" basal synaptic transmission.

Whatever the precise roles of LTP and LTD might be, the neural basis of learning will not be properly understood without full delineation of the contribution of generalized synaptic plasticity changes. Indeed, the influence of modulatory systems (e.g., cholinergic or monoaminergic) on learning might be on such generalized changes; the block of effects produced by muscarinic receptor antagonism in the present experiments could be produced in this way. An interesting question that arises from the current results is whether the effects on depotentiation and LTD might cause interference with subsequent discrimination learning. Future experiments are needed to address further this issue and the issue of specific as against generalized changes, because of its importance for the interpretation of experiments attempting to link synaptic plasticity mechanisms and learning.

\section{References}

Abraham WC, Robins A (2005) Memory retention-the synaptic stability versus plasticity dilemma. Trends Neurosci 28:73-78.

Abraham WC, Logan B, Greenwood JM, Dragunow M (2002) Induction and experience-dependent consolidation of stable long-term potentiation lasting months in the hippocampus. J Neurosci 22:9626-9634.

Aigner TG, Mishkin M (1993) Scopolamine impairs recall of one-trial stimulus reward association in monkeys. Behav Brain Res 54:133-136.

Anderson WW, Collingridge GL (2001) The LTP Program: a data acquisition program for on-line analysis of long-term potentiation and other synaptic events. J Neurosci Methods 108:71-83.

Bliss TVP, Collingridge GL (1993) A synaptic model of memory-long-term potentiation in the hippocampus. Nature 361:31-39.

Brown MW, Aggleton JP (2001) Recognition memory: what are the roles of the perirhinal cortex and hippocampus? Nat Rev Neurosci 2:51-61.

Brown MW, Bashir ZI (2002) Evidence concerning how neurons of the perirhinal cortex may effect familiarity discrimination. Phil Trans R Soc Lond B Biol Sci 357:1083-1095.

Brown MW, Xiang JZ (1998) Recognition memory: neuronal substrates of the judgement of prior occurrence. Prog Neurobiol 55:149-189.

Cho K, Kemp N, Noel J, Aggleton JP, Brown MW, Bashir ZI (2000) A new form of long-term depression in the perirhinal cortex. Nat Neurosci 3:150-156

Garden DL, Kemp N, Bashir ZI (2002) Differences in GABAergic transmission between two inputs into the perirhinal cortex. Eur J Neurosci 16:437-444.

Griffiths S, Scott H, Glover C, Bienemann A, Ghorbel MT, Uney J, Brown MW, Warburton EC, Bashir ZI (2008) Expression of long-term depression underlies visual recognition memory. Neuron 58:186-194.

Gruart A, Muñoz MD, Delgado-García JM (2006) Involvement of the CA3CA1 synapse in the acquisition of associative learning in behaving mice. J Neurosci 26:1077-1087.

Harder JA, Baker HF, Ridley RM (1998) The role of the central cholinergic projections in cognition: implications of the effects of scopolamine on discrimination learning by monkeys. Brain Res Bull 45:319-326.

Kemp N, Bashir ZI (2001) Long-term depression: a cascade of induction and expression mechanisms. Prog Neurobiol 65:339-365.

Kemp A, Manahan-Vaughan D (2007) Hippocampal long-term depression: master or minion in declarative memory processes? Trends Neurosci 30:111-118.

Malenka RC, Bear MF (2004) LTP and LTD: an embarrassment of riches. Neuron 44:5-21.

Manahan-Vaughan D, Braunewell K-H (1999) Novelty acquisition is associated with induction of hippocampal long-term depression. Proc Natl Acad Sci U S A 96:8739-8744.

Maren S (2005) Synaptic mechanisms of associative memory in the amyg dala. Neuron 47:783-786.

Martin SJ, Morris RGM (2002) New life in an old idea: the synaptic plasticity and memory hypothesis revisited. Hippocampus 12:609-636.

Massey PV, Bashir ZI (2007) Long-term depression: multiple forms and implications for brain function. Trends Neurosci 30:176-184.

Massey PV, Johnson BE, Moult PR, Auberson YP, Brown MW, Molnar E, Collingridge GL, Bashir ZI (2004) Differential roles of NR2A and NR2B-containing NMDA receptors in cortical long-term potentiation and long-term depression. J Neurosci 24:7821-7828.

Murray EA, Bussey TJ (1999) Perceptual-mnemonic functions of the perirhinal cortex. Trends Cogn Sci 3:142-151.

Murray WA, Richmond BJ (2001) Role of perirhinal cortex in object perception, memory, and associations Curr Opin Neurobiol 11:188-193.

Rioult-Pedotti MS, Friedman D, Hess G, Donoghue JP (1998) Strengthening of horizontal connections following skill learning. Nat Neurosci $1: 230-234$.

Rodrigues SM, Schafe GE, LeDoux JE (2004) Molecular mechanisms underlying emotional learning and memory in the lateral amygdala. Neuron 44:75-91.

Shors TJ, Matzel LD (1997) Long-term potentiation: what's learning got to do with it? Behav Brain Sci 20:597-614; discussion 614-655.

Wan H, Aggleton JP, Brown MW (1999) Different contributions of the hippocampus and perirhinal cortex to recognition memory. J Neurosci 19:1142-1148.

Warburton EC, Koder T, Cho K, Massey PV, Duguid G, Barker GRI, Aggleton 
JP, Bashir ZI, Brown MW (2003) Cholinergic neurotransmission is essential for perirhinal cortical plasticity and recognition memory. Neuron 38:987-996.

Warburton EC, Glover CPJ, Massey PV, Wan H, Johnson B, Bienemann A, Deuschle U, Kew JNC, Aggleton JP, Bashir ZI, Uney J, Brown MW (2005) cAMP responsive element-binding protein phosphorylation is necessary for perirhinal long-term potentiation and recognition memory. J Neurosci 25:6296-6303.

Whitlock JR, Heynen AJ, Shuler MG, Bear MF (2006) Learning induces long-term potentiation in the hippocampus. Science 313:1093-1097.

Wilson DA, Fletcher ML, Sullivan RM (2004) Acetylcholine and olfactory perceptual learning. Learn Mem 11:28-34.

Winters BD, Bussey TJ (2005) Removal of cholinergic input to perirhinal cortex disrupts object recognition but not spatial working memory in the rat. Eur J Neurosci 21: 2263-2270.
Xiang JZ, Brown MW (1998) Differential neuronal encoding of novelty, familiarity and recency in regions of the anterior temporal lobe. Neuropharmacology 37:657-676.

Xu L, Anwyl R, Rowan MJ (1998) Spatial exploration induces persistent reversal of long-term potentiation in rat hippocampus. Nature 394:891-894.

Zhu XO, McCabe BJ, Aggleton JP, Brown MW (1996) Mapping visual recognition memory through expression of the immediate early gene c-fos. Neuroreport 7:1871-1875.

Ziakopoulos Z, Tillett CW, Brown MW, Bashir ZI (1999) Input- and layerdependent synaptic plasticity in the rat perirhinal cortex in vitro. Neuroscience 92:459-472.

Ziakopoulos Z, Brown MW, Bashir ZI (2000) GABAB receptors mediate frequency-dependent depression of excitatory potentials in rat perirhinal cortex in vitro. Eur J Neurosci 12:803-809. 